\title{
Evaluation of the Tp-Te Interval, QTc and P-Wave Dispersion in Patients With Coronary Artery Ectasia
}

\author{
Ronan Conlon ${ }^{\mathrm{a}}$, Richard Tanner ${ }^{\mathrm{a}}$, Santhosh David ${ }^{\mathrm{a}}$, Gabor Szeplaki ${ }^{\mathrm{b}}$, Joseph Galvin ${ }^{\mathrm{b}}$, \\ John Keaney ${ }^{\mathrm{b}}$, Edward Keelan ${ }^{\mathrm{b}}$, Usama Boles ${ }^{\mathrm{a}, \mathrm{b}, \mathrm{c}}$
}

\begin{abstract}
Background: Coronary artery ectasia (CAE) is defined as a diffuse dilatation of the diameter of the ectatic segment of the coronary artery, 1.5 times greater than that of the adjacent segment. The Tp-Te interval, P-wave and QTc dispersions are relatively new electrocardiographic markers associated with an increased risk of developing arrhythmias. Despite CAE increasing in prevalence in recent years, there is a sparsity of data available about its arrhythmogenic effect. The aim of the study was to evaluate QTc, P-wave dispersion and Tp$\mathrm{Te}$ and $\mathrm{Tp}-\mathrm{Te} / \mathrm{QT}$ ratio in patients with $\mathrm{CAE}$.
\end{abstract}

Methods: A retrospective comparative study was designed for consecutive age- and sex-matched patients. Twenty patients with isolated CAE (group 1) and 20 control subjects (group 2), with normal coronary arteries, were included. All patients presented with chest pain and coronary angiogram was indicated. Outcome measures included $\mathrm{Tp}$-Te interval, Tp-Te/QT ratio, QTc dispersion and P-wave dispersion. Measurement of electrocardiogram (ECG) parameters was conducted using standardized digital online software. Descriptive and inferential statistics were performed.

Results: Mean Tp-Te $(95.5 \pm 9.01 \mathrm{~ms})$ and Tp-Te/QT ratio $(0.22 \pm$ 0.02 ) were significantly prolonged in CAE group (Tp-Te: $84 \pm 5.62$ $\mathrm{ms}, \mathrm{P}=0.00009$; $\mathrm{Tp}-\mathrm{Te} / \mathrm{QT}$ ratio: $0.20 \pm 0.01, \mathrm{P}=0.00004)$. In addition, QTc $(31.2 \pm 3.71 \mathrm{~ms})$ and $\mathrm{P}$-wave dispersion $(31.9 \pm 5.46 \mathrm{~ms})$ were significantly increased in comparison to the control group (QTc: $27.6 \pm 2.82 \mathrm{~ms}, \mathrm{P}=0.00532$ and $20 \pm 3.77 \mathrm{~ms}, \mathrm{P}=0.00003$ respectively). However, there was no difference in ventricular activation time (VAT) between groups.

Conclusions: CAE ECGs were found to be associated with increased $\mathrm{Tp}$-Te, Tp-Te/QT ratio, QTc intervals and P-wave dispersions. This may suggest that $\mathrm{CAE}$ existence has a pro-arrhythmogenic nature.

Keywords: Coronary artery ectasia; Tp-Te interval; QTc dispersions; Arrhythmia; P-wave dispersions; Tp-Te/QT ratio

Manuscript submitted November 10, 2017, accepted November 30, 2017

a Letterkenny University Hospital, Letterkenny, Co. Donegal, Ireland ${ }^{b}$ Heart and Vascular Center, Mater Private Hospital, Dublin 2, Ireland ${ }^{c}$ Corresponding Author: Usama Boles, Heart and Vascular Center, Mater Private Hospital, Dublin 2, Ireland. Email: bolesu@tcd.ie

doi: https://doi.org/10.14740/cr631w

\section{Introduction}

Coronary artery ectasia (CAE) has been defined dilatation of a coronary vessel segment up to 1.5 times the diameter of the adjacent vessel and including more than a third of the total length of the vessel [1]. The pathophysiology and clinical significance of CAE is poorly understood. Suggested etiologies include atherosclerosis, congenital abnormalities, inflammatory and/ or connective tissue diseases [2, 3].

The interval from the peak of the $T$ wave to the end of the $T$ wave (Tp-Te interval), on a standard 12-lead electrocardiogram (ECG), is a measure of transmural dispersion of repolarization in the left ventricle [4-6], with prolongation representation a period of possible susceptibility to ventricular arrhythmias [7, 8]. A prolonged Tp-Te interval has been associated with an increased risk of mortality in hypertrophic cardiomyopathy, in patients undergoing primary percutaneous coronary intervention for myocardial infarction, and in congenital and acquired longQT syndromes [9-11]. Limited data are available with regard to the effect that CAE has on the Tp-Te interval, which is important given its potential arrhythmogenic effect (Fig. 1).

Other ECG parameters such as QTc dispersion, which has been defined as the difference between the longest and shortest QT interval and P-wave dispersion (PWD), which is calculated by subtracting the minimum P-wave duration from the maximum in a 12-lead ECG (Fig. 1), are important measurements and have been implicated in the development of both ventricular and atrial arrhythmias respectively [12-14]. In the present study, we examine the Tp-Te interval, QTc and PWD in patients with CAE, to assess whether an apparently benign disease has a pro-arrhythmogenic effect.

\section{Materials and Methods}

A retrospective chart review of 1,650 consecutive angiograms, between January 2014 and January 2017, was conducted to identify patients with CAE [15]. Patients with known previous myocardial infarction (MI), atrial fibrillation, bundle branch blocks, ventricular preexcitation and atrioventricular conduction abnormalities were excluded from the study. Also, patients on any antiarrhythmic drugs that may have an effect on ECGs parameters were excluded. A total of 20 patients with isolated CAE (group 1) were identified and compared to 20 control subjects (group 2), with normal coronary arteries. All 


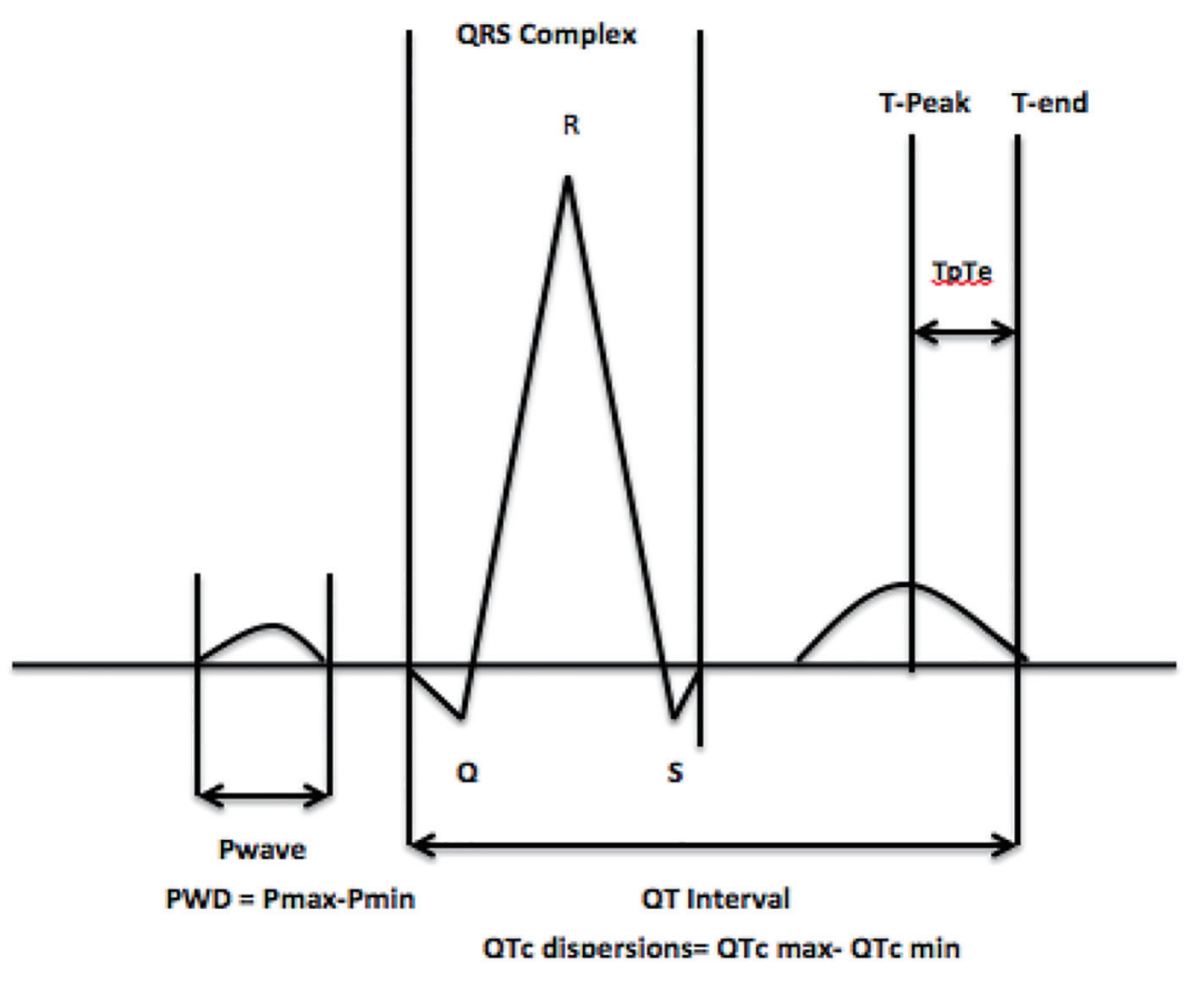

Figure 1. The Tp-Te interval, QTc and P-wave dispersion.

patients presented with chest pain and following non-invasive tests for risk stratification, for ischemic heart disease a coronary angiogram was indicated. Both groups were matched for age and sex as well as cardiovascular risk factors including: hypertension, family history of ischemic heart disease, diabetes mellitus, smoking history and dyslipidemia.

All ECGs were measured at rest, using a 12-lead high resolution ECG (GE, MAC 5000, New York) with $1 \mathrm{mV} / \mathrm{cm}$ amplitude and $25 \mathrm{~mm} / \mathrm{s}$ rate. Outcome measures included TpTe interval, Tp-Te/QT ratio, QTc dispersion, PWD and VAT.

\section{Tp-Te interval and $\mathrm{Tp}-\mathrm{Te} / \mathrm{QT}$ ratio}

The Tp-Te interval was measured from the peak of the $\mathrm{T}$ wave to the end of the $\mathrm{T}$ wave. The end of the $\mathrm{T}$ wave was defined as the intersection of the tangent to the down slope of the $T$ wave and the isoelectric line [16]. The QT interval was measured from the earliest onset of the QRS complex to the end of the T wave (Fig. 1). When a $U$ wave interrupted the $T$ wave prior to returning to baseline, the interval was measured to the nadir of the curve between the $T$ and $U$ waves.

The Tp-Te/QT ratio was calculated as the ratio of Tp-Te in lead V5 to the corresponding QT interval in the same lead [9]. The Tp-Te and QT interval were measured in lead V5 $[9,17]$.

\section{QTe dispersion}

QTc dispersion was defined as the difference between the long- est and shortest QT intervals and rate corrected using Bazett's formula, as the heart rate was found to be under $100 \mathrm{bpm}$ in all ECGs [12].

\section{PWD}

PWD was calculated by subtracting the minimum P-wave duration from the maximum in any of the 12 ECG leads (Fig. 1). The beginning of the $\mathrm{P}$ wave was defined as the point where the initial deflection of the $P$ wave crossed the isoelectric line and the end was defined as the point where the final deflection of the $\mathrm{P}$ wave crossed the isoelectric line.

\section{Ventricular activation time (VAT)}

VAT was defined as the average measurement from the onset of the $\mathrm{Q}$ wave to the peak of the $\mathrm{R}$ wave. The measurements from V6 were comparable with the mean value calculated from 12 leads as previously validated [18].

\section{ECG measurements}

Measurement of ECG parameters was conducted manually using digital online software (Cardio Calipers, Iconico, New York, NY), by a blinded observer. A second blinded observer repeated all ECG measurements using the same technique and 
Table 1. Demographics and Cardiovascular Risk Factors: Group 1 Versus Group 2

\begin{tabular}{llll}
\hline Variables & Group 1 (n= 20) & Group 2 $(\mathbf{n}=\mathbf{2 0})$ & P value \\
\hline Age & $63.2 \pm 10.2$ & $60 \pm 8.8$ & 0.58 \\
Gender (female, \%) & $14 / 6$ & $15 / 5$ & 0.77 \\
Hypertension (\%) & $12(60 \%)$ & $11(55 \%)$ & 0.31 \\
FHx of CAD (\%) & $6(30 \%)$ & $4(20 \%)$ & 0.71 \\
Diabetes mellitus (n, \%) & $4(20 \%)$ & $3(15 \%)$ & 0.40 \\
Smoking history (n, \%) & $14(70 \%)$ & $10(50 \%)$ & 0.94 \\
Dyslipidemia (n, \%) & $16(80 \%)$ & $13(65 \%)$ & 0.98 \\
\hline
\end{tabular}

FHx: family history; CAD: coronary artery disease.

the two subsets of data were compared to ensure there was no inter-observer variability $(\mathrm{P}>0.05)$.

\section{Statistical analysis}

Descriptive and inferential statistics were performed using Stata 13 (StataCorp CP, College Station, TX). An unpaired $t$ test was used to compare groups. Statistical significance was set at $\mathrm{P}<0.05$.

\section{Results}

\section{Demographics}

Baseline demographics of age, gender and cardiovascular risk factors including: hypertension, family history of ischemic heart disease, diabetes mellitus, smoking history and dyslipidemia were all listed in Table 1 . There was no significant difference between both groups. Hypertension was defined in accordance with the JNC-7 guidelines as a systolic blood pressure $\geq 140 \mathrm{~mm} \mathrm{Hg}$, diastolic blood pressure $\geq 90 \mathrm{~mm} \mathrm{Hg}$ and/or use of antihypertensive medications. Family history of CAD was noted if a male first-degree relative developed CAD before the age of 55 or a female first-degree related before 65 . Diabetes was defined as overnight fasting blood glucose $\geq 7$ $\mathrm{mmol} / \mathrm{L}(126 \mathrm{mg} / \mathrm{dL})$, postprandial blood glucose $\geq 11 \mathrm{mmol} / \mathrm{L}$ (200 mg/dL) or use of insulin or oral hypoglycemic agents. Patients were classified as smokers if they had smoked during the last month. Dyslipidemia was defined as a total cholesterol $\geq$
$6.2 \mathrm{mmol} / \mathrm{L}(240 \mathrm{mg} / \mathrm{dL})$, low-density lipoprotein cholesterol $\geq 4.14 \mathrm{mmol} / \mathrm{L}(160 \mathrm{mg} / \mathrm{dL})$ or use of lipid-lowering medications [19].

\section{CAE/ECG comparison with control}

In Table 2, all the predefined intervals were compared and showed results as follows.

\section{Tp-Te interval and Tp-Te/QT ratio}

Mean Tp-Te $(95.5 \pm 9.01 \mathrm{~ms})$ and Tp-Te/QT ratio $(0.22 \pm 0.02)$ were significantly prolonged in group 1, compared to group 2 (Tp-Te: $84 \pm 5.62 \mathrm{~ms}, \mathrm{P}=0.00009$; Tp-Te/QT ratio: $0.20 \pm$ $0.01, \mathrm{P}=0.00004)$.

\section{QTc dispersion and PWD}

CAE/ECG measurements of QTc $(31.2 \pm 3.71 \mathrm{~ms})$ and PWD $(31.9 \pm 5.46 \mathrm{~ms})$ were significantly higher in comparison to group 2 (QTc: $27.6 \pm 2.82 \mathrm{~ms}, \mathrm{P}=0.00532$; PWD: $20 \pm 3.77$ $\mathrm{ms}, \mathrm{P}=0.00003)$.

$V A T$

There was no significant difference in VAT duration between groups (group 1: $42 \pm 2.63 \mathrm{~ms}$, group 2: $43.72 \pm 3.23 \mathrm{~ms}, \mathrm{P}=$ $0.22)$.

Table 2. Comparison of ECG Intervals Between CAE Group and Control Group

\begin{tabular}{llll}
\hline Variables & CAE group $(\mathbf{n}=\mathbf{2 0})$, mean \pm SD & Control group $(\mathbf{n}=\mathbf{2 0})$, mean \pm SD & P value \\
\hline Tp-Te $(\mathrm{ms})$ & $95.5 \pm 9.01$ & $84 \pm 5.62$ & $<0.001$ \\
Tp-Te/QT ratio & $0.22 \pm 0.02$ & $0.20 \pm 0.01$ & $<0.001$ \\
QTc dispersions (ms) & $31.2 \pm 3.71$ & $27.6 \pm 2.82$ & $<0.01$ \\
P-wave dispersions (ms) & $31.9 \pm 5.4$ & $20 \pm 3.77$ & $<0.001$ \\
VAT (ms) & $42 \pm 2.63$ & $43.72 \pm 3.23$ & 0.22 \\
\hline
\end{tabular}

Tp-Te: peak T wave duration to the end of T wave; QTc: corrected QT dispersions; VAT: ventricular activation time. 


\section{Discussion}

The present study represents an assessment of pertinent ECG markers associated with arrhythmias in patients with CAE. CAE was found to be associated with a prolonged Tp-Te interval, QTc and PWD. These findings suggest that CAE, an apparently benign disease, may in fact have a pro-arrhythmogenic effect.

CAE is a relatively rare coronary anomaly, found in approximately $5 \%$ of patients undergoing coronary angiography [2]. Ectatic coronary arteries have been found to be associated with myocardial ischemia, thrombosis, spasm, dissection and rupture [20-22]. In addition, they have been associated with electrical instability, ventricular fibrillation and sudden death $[22,23]$.

\section{Tp-Te interval}

Tp-Te interval is a measure of transmural dispersion of repolarization in the left ventricle, with prolongation representing a period of possible susceptibility to ventricular arrhythmias [7, 8]. A proposed mechanism for this phenomenon was suggested by Antzelevitch and coworkers [24, 25]. Three electrophysiological distinct cell types exist in the ventricular myocardium, which include the endocardial, epicardial and subendocardial $\mathrm{M}$ cells (Masonic mid-myocardial Moe cells). The action potential of the $\mathrm{M}$ cells is the most susceptible to prolongation [6], likely because of a combination of larger late-sodium and sodium/calcium exchange currents as well as a weaker slowly activation delayed rectifier current [26]. The Tp-Te interval corresponds with transmural dispersion of repolarization in the ventricular myocardium, a period where the epicardium has fully repolarized but the $\mathrm{M}$ cells are still in the process of repolarization, making them more vulnerable to early after-depolarizations $[16,17]$. These early after-depolarizations can lead to reentry, which could result in a polymorphic ventricular tachycardia or ventricular fibrillation, hence why a prolonged Tp-Te likely corresponds to an extended period of vulnerability that could increase the risk of ventricular arrhythmogenesis [7, 27-29].

In the present study, the Tp-Te interval was found to be longer in the CAE group. Our results were consistent with another study conducted by Karaagac et al [30], where the TpTe interval was found to be significantly increased in patients with isolated CAE. In their study, the mean Tp-Te interval was found to be $97.71 \pm 8.7 \mathrm{~ms}$ in patients with CAE, compared to $85.23 \pm 7.1 \mathrm{~ms}$ in the group with normal coronary arteries [30].

A disproportionate increase in transmural dispersion of repolarization relative to the total duration of repolarization (Tp-Te/QT ratio) has previously been shown to have a proarrhythmic effect [31]. It has been proposed as a marker of arrhythmogenesis for long QT-syndrome as well as hypertrophic cardiomyopathy $[9,32]$. In the present study, mean Tp-Te/QT ratio was significantly greater among patients with CAE compared to controls.

\section{QTe dispersion}

QTe dispersion is emerging as an important clinical tool as it is known to be an important factor in the development of ventricular arrhythmias in patients with chronic heart failure, mitral valve prolapse, MI and familial long QT syndrome [13, 33-35]. In the present study, isolated CAE was associated with a significantly increased QTc in comparison to the control group. Our findings are consistent with another study, which also found QTc dispersion to be increased in patients with CAE [36].

\section{PWD}

PWD is thought to reflect prolongation of both intra-artial and interatrial conduction time and the inhomogeneous propagation of sinus impulses, which are known electrophysiological features in patients with atrial arrhythmias [14]. A prolonged PWD time has been reported to increase the risk of developing atrial fibrillation in patients with no underlying heart disease $[37,38]$. Our results were consistent with another study, which demonstrated a statistically significant increase in PWD time in patients with CAE [36].

\section{Conclusions}

CAE was found to be associated with prolongation of the TpTe interval, QTc and PWD. Our data suggest that these patients may be more susceptible to developing both ventricular and atrial arrhythmias. Routine measurement of the aforementioned ECG parameters in clinical practice may be useful in risk stratifying these patients. Further prospective studies are warranted to quantify arrhythmia burden in CAE patients.

\section{References}

1. Yetkin E, Ozturk S. Coronary artery aneurysm and coronary artery ectasia: what makes the difference? Angiology. 2017;68(9):833.

2. Demopoulos VP, Olympios CD, Fakiolas CN, Pissimissis EG, Economides NM, Adamopoulou E, Foussas SG, et al. The natural history of aneurysmal coronary artery disease. Heart. 1997;78(2):136-141.

3. Williams MJ, Stewart RA. Coronary artery ectasia: local pathology or diffuse disease? Cathet Cardiovasc Diagn. 1994;33(2):116-119.

4. Taggart P, Sutton PM, Opthof T, Coronel R, Trimlett R, Pugsley W, Kallis P. Transmural repolarisation in the left ventricle in humans during normoxia and ischaemia. Cardiovasc Res. 2001;50(3):454-462.

5. Opthof T, Coronel R, Janse MJ. Is there a significant transmural gradient in repolarization time in the intact heart?: Repolarization Gradients in the Intact Heart. Circ Arrhythm Electrophysiol. 2009;2(1):89-96.

6. Antzelevitch C, Sicouri S, Litovsky SH, Lukas A, Krishnan SC, Di Diego JM, Gintant GA, et al. Heterogeneity within the ventricular wall. Electrophysiology and pharmacology of epicardial, endocardial, and $\mathrm{M}$ cells. Circ Res. 1991;69(6):1427-1449. 
7. Yan GX, Antzelevitch C. Cellular basis for the normal T wave and the electrocardiographic manifestations of the long-QT syndrome. Circulation. 1998;98(18):1928-1936.

8. Antzelevitch C, Shimizu W, Yan GX, Sicouri S. Cellular basis for QT dispersion. J Electrocardiol. 1998;30(Suppl):168-175.

9. Shimizu M, Ino H, Okeie K, Yamaguchi M, Nagata M, Hayashi K, Itoh $\mathrm{H}$, et al. T-peak to T-end interval may be a better predictor of high-risk patients with hypertrophic cardiomyopathy associated with a cardiac troponin I mutation than QT dispersion. Clin Cardiol. 2002;25(7):335339.

10. Haarmark C, Hansen PR, Vedel-Larsen E, Pedersen SH, Graff C, Andersen MP, Toft E, et al. The prognostic value of the Tpeak-Tend interval in patients undergoing primary percutaneous coronary intervention for ST-segment elevation myocardial infarction. J Electrocardiol. 2009;42(6):555-560.

11. Topilski I, Rogowski O, Rosso R, Justo D, Copperman Y, Glikson M, Belhassen B, et al. The morphology of the QT interval predicts torsade de pointes during acquired bradyarrhythmias. J Am Coll Cardiol. 2007;49(3):320-328.

12. Ahnve S. Correction of the QT interval for heart rate: review of different formulas and the use of Bazett's formula in myocardial infarction. Am Heart J. 1985;109(3 Pt 1):568-574.

13. Barr CS, Naas A, Freeman M, Lang CC, Struthers AD. QT dispersion and sudden unexpected death in chronic heart failure. Lancet. 1994;343(8893):327-329.

14. Okutucu S, Aytemir K, Oto A. P-wave dispersion: What we know till now? JRSM Cardiovasc Dis. 2016;5:2048004016639443.

15. Boles U, Rakhit R, Shiu MF, Patel K, Henein M. Coronary artery ectasia as a culprit for acute myocardial infarction: review of pathophysiology and management. Anadolu Kardiyol Derg. 2013;13(7):695-701.

16. Perkiomaki JS, Koistinen MJ, Yli-Mayry S, Huikuri HV. Dispersion of QT interval in patients with and without susceptibility to ventricular tachyarrhythmias after previous myocardial infarction. J Am Coll Cardiol. 1995;26(1):174-179.

17. Bazett HC. An analysis of the time-relations of electrocardiograms. Heart. 1920;7:353-357.

18. Boles U, Almuntaser I, Brown A, Murphy RR, Mahmud A, Feely J. Ventricular activation time as a marker for diastolic dysfunction in early hypertension. Am J Hypertens. 2010;23(7):781-785.

19. Nicoll R, Wiklund U, Zhao Y, Diederichsen A, Mickley H, Ovrehus K, Zamorano P, et al. The coronary calcium score is a more accurate predictor of significant coronary stenosis than conventional risk factors in symptomatic patients: Euro-CCAD study. Int J Cardiol. 2016;207:13-19.

20. Suzuki H, Takeyama Y, Hamazaki Y, Namiki A, Koba $\mathrm{S}$, Matsubara H, Hiroshige $\mathrm{J}$, et al. Coronary spasm in patients with coronary ectasia. Cathet Cardiovasc Diagn. 1994;32(1):1-7.

21. Perlman PE, Ridgeway NA. Thrombosis and anticoagulation therapy in coronary ectasia. Clin Cardiol. 1989;12(9):541-542.
22. Satoda M, Tatsukawa H, Katoh S. Images in cardiovascular medicine. Sudden death due to rupture of coronary aneurysm in a 26-year-old man. Circulation. 1998;97(7):705-706.

23. Karakaya O, Saglam M, Barutcu I, Esen AM, Turkmen M, Kargin R, Esen O, et al. Effects of isolated coronary artery ectasia on electrocardiographic parameters reflecting ventricular heterogeneity. J Electrocardiol. 2007;40(2):203-206.

24. Antzelevitch CSW, Yan GX. Electrical heterogeneity and the development of arrhythmias. In: Olsson SB, Yuan S, Amlie JP, editors. Dispersion of ventricular repolarization:state of the art. Armonk (NY): Futura Publishing Company; 2000. p. 3.

25. Sicouri S, Antzelevitch C. A subpopulation of cells with unique electrophysiological properties in the deep subepicardium of the canine ventricle. The M cell. Circ Res. 1991;68(6):1729-1741.

26. Antzelevitch C. Role of spatial dispersion of repolarization in inherited and acquired sudden cardiac death syndromes. Am J Physiol Heart Circ Physiol. 2007;293(4):H2024-2038.

27. Antzelevitch C, Shimizu W, Yan GX, Sicouri S, Weissenburger J, Nesterenko VV, Burashnikov A, et al. The M cell: its contribution to the ECG and to normal and abnormal electrical function of the heart. J Cardiovasc Electrophysiol. 1999;10(8):1124-1152.

28. Emori T, Antzelevitch C. Cellular basis for complex $\mathrm{T}$ waves and arrhythmic activity following combined $\mathrm{I}(\mathrm{Kr})$ and $\mathrm{I}(\mathrm{Ks})$ block. J Cardiovasc Electrophysiol. 2001;12(12):1369-1378.

29. Watanabe N, Kobayashi Y, Tanno K, Miyoshi F, Asano T, Kawamura M, Mikami Y, et al. Transmural dispersion of repolarization and ventricular tachyarrhythmias. J Electrocardiol. 2004;37(3):191-200.

30. Karaagac K, Yontar OC, Tenekecioglu E, Vatansever F, Ozluk OA, Tutuncu A, Yagcioglu P, et al. Evaluation of Tp-Te interval and Tp-Te/QTc ratio in patients with coronary artery ectasia. Int J Clin Exp Med. 2014;7(9):28652870.

31. Gupta P, Patel C, Patel H, Narayanaswamy S, Malhotra B, Green JT, Yan GX. T(p-e)/QT ratio as an index of arrhythmogenesis. J Electrocardiol. 2008;41(6):567-574.

32. Yamaguchi $M$, Shimizu M, Ino H, Terai H, Uchiyama K, Oe K, Mabuchi T, et al. T wave peak-to-end interval and QT dispersion in acquired long QT syndrome: a new index for arrhythmogenicity. Clin Sci (Lond). 2003;105(6):671676.

33. Tieleman RG, Crijns HJ, Wiesfeld AC, Posma J, Hamer HP, Lie KI. Increased dispersion of refractoriness in the absence of QT prolongation in patients with mitral valve prolapse and ventricular arrhythmias. Br Heart J. 1995;73(1):37-40.

34. Higham PD, Furniss SS, Campbell RW. QT dispersion and components of the QT interval in ischaemia and infarction. Br Heart J. 1995;73(1):32-36.

35. Day CP, McComb JM, Campbell RW. QT dispersion: an indication of arrhythmia risk in patients with long QT intervals. Br Heart J. 1990;63(6):342-344. 
36. Mahmoud K. Effect of isolated coronary artery ectasia on dispersion of P-wave and QT interval. The Egyptian Heart Journal. 2012;64:121-125.

37. Dilaveris PE, Gialafos EJ, Sideris SK, Theopistou AM, Andrikopoulos GK, Kyriakidis M, Gialafos JE, et al. Simple electrocardiographic markers for the prediction of paroxysmal idiopathic atrial fibrillation. Am Heart J. 1998;135(5 Pt 1):733-738.

38. Gialafos JE, Dilaveris PE, Gialafos EJ, et al. P dispersion: a valuable electrocardiographic marker for the prediction of paroxysmal lone atrial fibrillation. Ann Noninvasive Electrocardiol. 1999;4:39-45. 